\title{
A revival of electric analogs for vibrating mechanical systems aimed to their efficient control by PZT actuators
}

\author{
Silvio Alessandroni ${ }^{\text {a }}$, Francesco dell'Isola ${ }^{\mathrm{b}, *}$, Maurizio Porfiri ${ }^{\mathrm{c}}$ \\ a Dottorato in Meccanica teorica ed applicata, Università di Roma "La Sapienza", 00184 Roma, Italy \\ b Dipartimento di Ingegneria Strutturale e Geotecnica, Università "La Sapienza", 00184 Roma, Italy \\ ${ }^{c}$ Department of Engineering Science and Mechanics, Virginia Polytechnic Institute and State University, \\ Blacksburg, VA 24061-0219, USA
}

Received 8 September 2001

\begin{abstract}
In this paper we revive classical methods (i.e. those needed to synthesize analog circuits for designing direct computers) in order to conceive novel devices which we call piezo-electro-mechanical (PEM) structural members. By means of two independent synthesis procedures, new circuital analogs for the uniformly damped Elastica and Kirchhoff-Love plate are found. These circuits are used as electrical wave guides gyroscopically coupled to the corresponding mechanical members to design electrically dissipative PEM systems. The concept on which these systems are based exploits piezoelectric transducers - uniformly distributed on the member-to transform strain energy into capacitive energy: this last will be subsequently dissipated using resistors, the optimal value of which is determined by a pole placement criterion. We underline that in PEM structures PZT transducers can be regarded, at the same time, as sensors and actuators. By means of analytical methods and numerical simulations, the electro-mechanical constitutive parameters of some PEM structures are determined and it is shown that they can be designed and may be technically feasible. Moreover it is shown that in PEM structures, also when mechanical dissipative phenomena are negligible, mechanical vibrations are efficiently damped by means of completely passive electrical circuits.
\end{abstract}

(C) 2002 Published by Elsevier Science Ltd.

Keywords: Mechanical vibrations control; Piezoelectric distributed control; Electro-mechanical dampers

An analogy is a recognized relationship of consistent mutual similarity between the equations and structures appearing within two or more fields of knowledge, and an identification and association of the quantities and structural elements which play mutually similar roles in these equations and structures, for the purpose of facilitating transfer of knowledge of mathematical procedures of analysis and behavior of the structures between these fields (Firestone, 1956).

\footnotetext{
${ }^{*}$ Corresponding author.

E-mail address: francesco.dellisola@uniroma1.it (F. dell'Isola).
} 


\section{Introduction}

\subsection{Objectives}

In this paper well-established methods (i.e. those needed to synthesize the analog circuit for designing direct computers) are revived in order to conceive novel devices: i.e. piezo-electro-mechanical structural members. These devices are conceived as structural members in which a structural modification is introduced aiming to obtain an efficient mechanical vibration damping. The structural modification consists in bonding PZT transducers to the structural member and in electrically interconnecting them by means of suitably synthesized circuits. We explicitly state that:

1. The interconnecting circuits must be governed by equations which are analogous to those governing the mechanical vibrations.

2. We could not find anywhere in the literature the circuits which we have synthesized and are described in the paper.

For an exhaustive treatment of the state of the art in analog methods we refer to Karplus and Soroka (1959), Brillouin (1946) and Crandall et al. (1968).

The behavior of PEM structures and their properties are investigated by means of analytical methods and numerical simulations: we prove that, once the mechanical properties of the structure are known, the interconnecting electrical circuits can be designed in order to optimize its mechanical damping.

\subsection{Historical background}

A first attempt to exploit the direct and inverse piezoelectric effect in the conception of an electromechanical transducer dates up to the late sixties (for reference see for e.g. Bardati et al., 1968; Barzilai and Gerosa, 1967): such a device was imagined essentially constituted by two layers of deformable materials, one of which exhibiting the behavior of a piezo-dielectric. The expected effect of the electromagnetic wave was the deformation of the materials so to produce a mechanical wave.

Unfortunately it can be proven (see Bardati et al., 1968) that such a device cannot work: this is mainly due to the huge difference between the electromagnetic and mechanical wave speeds. This impossibility result made clear that only quasi-static electromagnetic regimes needed to be considered in the conception of lumped energy transducers based on the piezoelectric effect. Therefore Engineering Science addressed research efforts towards the realization of "quasi-static" piezoelectric actuators driven by "slowly varying" electric fields.

The most recent technological developments endowed "quasi-static" piezoelectric actuators with highelectro-mechanical coupling capability and more performing mechanical properties. Therefore an increasing interest in mechanical vibrations control exploiting piezoelectric effect aroused. Indeed presently (see ACX and Piezo Systems) comparatively very efficient and reliable "capacitive" PZT actuators are designed and produced: they are able to exert up to hundreds of newton, in presence of elongations of hundreds of microstrains and applied voltage of hundreds of volt.

The behavior of such electro-mechanical transducers has been extensively studied (see Rogacheva, 1994; Gandhi and Thompson, 1992; Inman, 2000; ACX). They show a two-fold behavior: as mechanical devices they add stiffness to the structure and exert a mechanical action expending power on the deformation rate, while, regarded as electrical devices, their Norton equivalent representation is a capacitor in parallel connection with a current generator. The exerted mechanical action and the current generator are driven respectively by the actuator capacitive voltage and deformation rate so that the whole device can be regarded as loss less. 
In the literature, using these actuators (see e.g. Wang, 1995; Valis et al., 1992), it is studied a lumped control method for a single beam via one of the described piezoelectric actuators properly placed in the area of highest strain and connected to an electric circuit resonant at a given eigenfrequency of the beam itself.

As the theorem of maximum power transfer holds (see e.g. Balanis, 1997), this apparatus can allow for a non-negligible energy transfer between the mechanical and the electrical forms, once the electrical impedance is adapted to the mechanical one so that an electro-mechanical resonance is established and the coupling effect, assured by the piezoelectric actuator, is effective.

When a set of actuators is used, the problem of their optimal location on the structural member arises. Obviously such a location strongly depends on the mode to be controlled: indeed in both the case of beam or plate vibrations control it can be proven (see e.g. Batra and Ghosh, 1995) that a location effective for one mode is often ineffective for the others. Therefore several authors (see e.g. Bernadou and Haenel, 2000; Bernadou and Haenel, submitted; Hoffmann and Botkin, 1999; Botkin, 1999; Hoffmann and Botkin, 2000) considered plates in which a set of piezoelectric actuators is uniformly embedded. The homogenized evolution equations for such a device are derived when (i) the number of actuators is very large, their size is correspondingly small and the plate area "covered" by the actuators is kept fixed (ii) every actuator is connected to an electric circuit imposing at its electric terminals a given potential drop, eventually depending on time. In all described devices each actuator is electrically connected to a single circuit which has to include active components, also when the circuit is a (nominally passive) resonant RLC: indeed the requested inductances are too high to be technically feasible without voltage power supplies. Furthermore using the single-actuator-connected-to-a-single-resonant-circuit configuration, every actuator could be forced in some particular circumstances to stand a greater amount of the electro-mechanical energy transfer from the vibrating beam to the resonant circuit. As a consequence high performances are required to the single actuator.

In order to cure these drawbacks in dell'Isola and Vidoli (1998a,b) it is proposed to interconnect a set of piezoelectric actuators - uniformly distributed over a beam - to a lumped circuit so as to obtain a transmission line. In this way it is provided a support for the propagation of an electric wave, the speed of which can be suitably tuned to the mechanical wave speed. This concept again is related to the result described by Bardati et al. (1968) and Barzilai and Gerosa (1967): indeed as the electromagnetic waves propagate in a dielectric layer too quickly then, in order to have an efficient electro-mechanical energy transfer, such a layer has to be split into a set of lumped piezoelectric actuators "electrically embedded" in a waveguide synthesized so to get low-speed waves.

Such a propagative approach was explicitly proposed and studied by dell'Isola and Vidoli (1998a,b), where an electro-mechanical waveguide with the electric wave component not directly supported by a piezoelectric layer is considered.

A precursory paper to dell'Isola and Vidoli (1998a,b) is Hagood and von Flotow (1991) where it is experimentally proven that a bending vibrating beam can excite a "telegraphist" lumped circuit waveguide.

The notion of electro-mechanical coupled wave propagation seems very fruitful in the design of devices aimed to control mechanical vibration using piezoelectric actuators: indeed it requires less performing actuators and electric components, distributing the coupling tasks among several piezoelectric patches which respond synergically, (see dell'Isola and Vidoli, 1998a,b; Vidoli and dell'Isola, 2000; Valis et al., 1992). In dell'Isola and Vidoli (1998a,b) it is seen that because of the analogy between the telegraphist transmission line and the extensionally vibrating beam, a simultaneous control of all the extensional vibrating modes can be achieved, while such a simultaneous control cannot be extended to the transverse vibration of the beam; in particular the considered transmission line can be tuned to control only one transversal mode of the system. This limit is recognized to be related (Vidoli and dell'Isola, 2000) to the different spectral properties of the operators governing the deflection of the transversely vibrating beam and the electric signal in the telegraphist transmission line. 


\subsection{Description of the proposed novel device}

The present paper stems from some results found in Vidoli and dell'Isola (2000): they indicate that in order to obtain the most efficient way to control vibration in a given frame, via piezoelectric actuator interconnected to electric circuits, modal characteristics must coincide with those of the electrical network.

We find some analog circuits for the Euler beam and Kirchhoff-Love plate also in presence of dampers. We then draw our attention to what we call conservative piezo-electro-mechanical systems (or conservative PEM structures).

They are constituted by:

1. a structural member (e.g. a plate or a beam, see for instance Fig. 1),

2. a set of actuators uniformly distributed on the considered structural member,

3. the corresponding analog circuit including as elements the piezoelectric actuator.

As the main purpose of our design is to conceive a device apt to efficiently damp structural vibrations, we also introduce the "electrically dissipative" PEM structures.

They are realized coupling (by means of piezoelectric actuators) the mechanical structure to be damped to the electric analog of the same structure but embedded in a viscous fluid (or equivalently connected to a "bed" of dampers).

Some initial and boundary value problems for electrically dissipative PEM structures are solved using Galerkin method, and a complete free propagation analysis for conservative PEM beams is developed.

The solutions we have obtained allow us to state that:

1. There exists an optimal circuital resistance enabling an efficient damping of mechanical vibration. Although this resistance depends on the mode, that adapted for the first one is effective for all the others.

2. The concept underlying PEM structures seems to be ready for technological applications. Indeed we establish general design procedures for the considered electro-mechanical structures and prove that the commercialized piezoelectric actuators allow for the constructions of technologically relevant devices. The preparation of an experimental set-up seems to be feasible.

3. We prove that PEM dissipative structures represent a technologically effective structural modification of the original mechanical ones (for a detailed discussion about the concept of structural modification see e.g. D'Ambrogio and Sestieri, 1989). Indeed the introduced dissipative devices, while being effective, do not impose constraints on the design and performances of mechanical structures to be damped.

\subsection{Synopsis}

In Section 2 different approaches to the synthesis of electric analog for a transversely vibrating beam are addressed, providing circuits suitable to different fields of applications. We extend the results found in

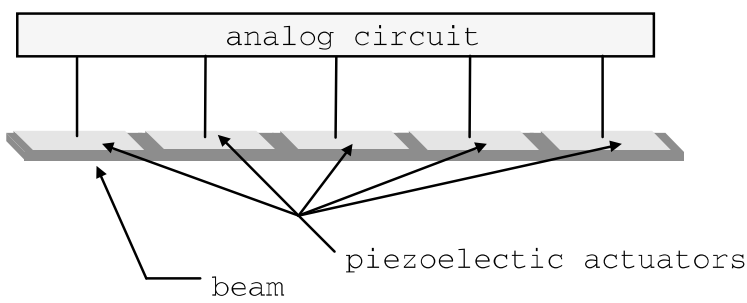

Fig. 1. Sketch of a PEM beam. 
Molloy (1958) generalizing the mobility representation to multiterminal structures and their synthesis techniques by the introduction of multiport networks (Newcomb, 1966). The sophisticated synthesis procedure we have used allows for the synthesis of networks constituted by only purely passive and technically realizable components. The developed space discretization schemes parallel the time discretization ones, of importance in the design of digital filters employed e.g. in the control of industrial machines (see e.g. Papoulis, 1991).

In Section 3 the previously derived electric analogs are coupled to a beam frame via piezoelectric actuators uniformly distributed on the structural members. Introducing a homogenized mathematical model for PEM structures, a formal analysis of the coupled vibrating system is exhibited, supported by numerical simulations. A more detailed study of simply supported or infinite PEM beams concludes the section.

In Section 4 resistors are properly inserted in the conservative analog circuits in order to get a purely electrical damper. In a specific set of circuital analogs an optimal damping resistance is obtained using the methods of Theory of Control.

In Section 5 an extension of the previous results obtained for a beam is provided for a plate.

\section{Electrical analogs}

\subsection{The Euler beam}

In order to derive the analog circuit for the Elastica, we will briefly recall a dimensionless form for its balance and constitutive equations.

Let us consider a Saint-Venant purely flexible beam: the length of its axis will be denoted by $l$, the thickness of its section by $h$ (assumed to be rectangular), its bending stiffness and mass density per unit length by $K_{M}{ }^{1}$ and $\rho$.

We choose $l$, a given fixed moda circularl frequency ${ }^{2} \varpi$ and $h$ as the characteristic constants for the dimensionless abscissa $x$, Laplace variable $s$ and deflection $u$ respectively. If rotational inertia can be neglected, the dimensionless couple and force balance equations are (the superscript $\dot{\square}$ represents the derivative with respect to the dimensionless time $t$ ):

$$
\begin{aligned}
& M^{\prime}(x, t)+T(x, t)=0, \\
& T^{\prime}(x, t)=\frac{l h \varpi^{2}}{T_{0}} \rho \ddot{u}(x, t),
\end{aligned}
$$

where $M$ and $T$ are the dimensionless contact actions, and the characteristic bending moment $M_{0}$ and shear contact force $T_{0}$ are chosen as follows:

$$
\begin{aligned}
& M_{0}=\frac{h}{l^{2}} K_{M}, \\
& T_{0}=\frac{h}{l^{3}} K_{M} .
\end{aligned}
$$

Moreover the constitutive equation for the dimensionless bending moment $M$ becomes (the superscript $\square^{\prime}$ represents the dimensionless spatial derivative):

$$
M(x, t)=u^{\prime \prime}(x, t)
$$

\footnotetext{
${ }^{1} K_{M}=Y I$, where $Y$ is the Young modulus of the material constituting the beam and $I$ is the moment of inertia of the beam section.

${ }^{2}$ The characteristic time is consequently chosen as the inverse of $\varpi$.
} 
Finally the governing equation for the deflection velocity $v$ becomes:

$$
v^{\mathrm{IV}}(x, t)+\alpha^{4} \ddot{v}(x, t)=0,
$$

with

$$
\alpha^{4}=\varpi^{2} l^{4} \frac{\rho}{K_{M}} .
$$

It is convenient for our purposes to consider the problem in the Laplace domain, so that (1) becomes (where

$\widetilde{d e n o t e s}$ the Laplace transform, and $s$ represents the dimensionless Laplace variable):

$$
\begin{aligned}
& \tilde{M}^{\prime}(x, s)+\tilde{T}(x, s)=0, \\
& \tilde{T}^{\prime}(x, s)=\alpha^{4} s^{2} \tilde{u}(x, s),
\end{aligned}
$$

and the governing equation for the deflection velocity $v$ in the Laplace domain is:

$$
\tilde{v}^{\mathrm{IV}}(x, s)+\alpha^{4} s^{2} \tilde{v}(x, s)=0 .
$$

\subsection{Lumped circuital analogs for the elastica}

In order to synthesize electrical analogs for the Elastica, different procedures can be used, each of them leading, in general, to a particular circuital realization.

Indeed we can exhibit two different discrete forms of Eq. (7), defined on a set of sampling nodes equally spaced on the beam (as shown in Fig. 2), to each of which different circuital graphs can be associated.

The first form is derived directly replacing the spatial differential operator in (7) by its centered finite difference approximation, while the second one is based upon an approximated solution of a normal system associated to (7).

The lumped circuits synthesized by the two techniques are different: the first one shows a simpler topology (easily generalized to a plate), even if it cannot dispense with a power supply; while the second one is constituted by completely passive electric components, even if it shows a more elaborate topology.

The flow chart in Fig. 3 illustrates the different paths walked in the two discussed approaches.

\subsubsection{Synthesis of the analog circuit based on the finite difference method}

Considering a centered finite difference rule, the discrete form of (7) becomes:

$$
\frac{\tilde{v}_{k-2}(s)-4 \tilde{v}_{k-1}(s)-4 \tilde{v}_{k+1}(s)+\tilde{v}_{k+2}(s)}{\delta^{4}}+\left(\alpha^{4} s^{2}+\frac{6}{\delta^{4}}\right) \tilde{v}_{k}(s)=0,
$$

where $k$ labels a generic inner node of the mesh (the boundary nodes will be separately discussed according to the particular constraints imposed on the beam).

Let us explicitly remark that (8) represents a set of algebraic equations, which we want to regard as the set of current balance equations for the sampling electric nodes of the analog circuit, once each node of the electrical circuit is chosen to correspond to a unique sampling spatial node.

The electrical kinematical descriptor chosen to synthesize the analog is the voltage drop between each sampling node and the ground. This choice corresponds to associate the dimensionless deflection velocity $v_{k}$

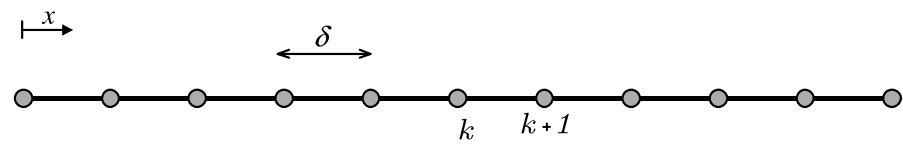

Fig. 2. Mesh on the beam. 


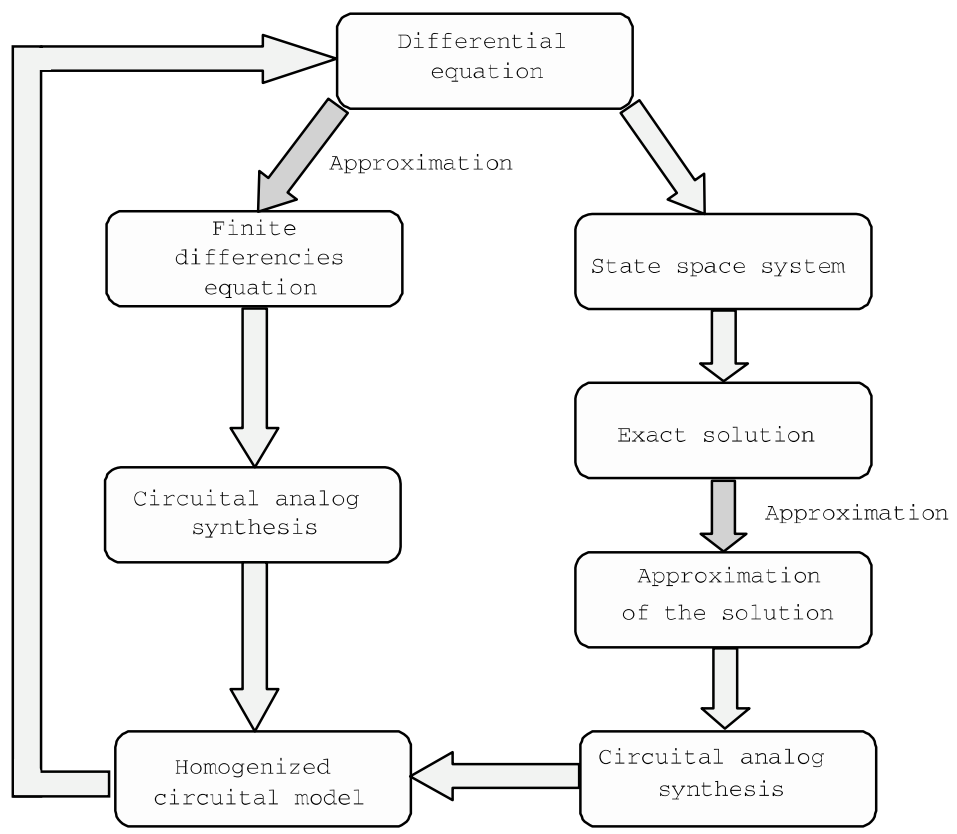

Fig. 3. Flow chart illustrating the different strategies to the solution of the synthesis problem.

at each sampling node on the beam to the dimensionless potential $V_{k}$ at the corresponding electric node of the analog circuit. ${ }^{3}$

Our goal is to find a circuital topology, so that the current balance equation at each node is

$$
\frac{\tilde{V}_{k-2}(s)-4 \tilde{V}_{k-1}(s)-4 \tilde{V}_{k+1}(s)+\tilde{V}_{k+2}(s)}{\delta^{4}}+\left(\alpha^{4} s^{2}+\frac{6}{\delta^{4}}\right) \tilde{V}_{k}(s)=0 .
$$

The branches of the sought circuital topology are assumed to be constituted by only two terminal networks so that the current flowing through each circuital branch depends only on the voltage drop between the connected nodes. These assumptions suggest to connect the $k$ th node as shown in Fig. 4, i.e.:

1. to the ground,

2. to the $(k \pm 1)$ th nodes and

3 . to the $(k \pm 2)$ th nodes.

Assuming that (9) is obtained as the current balance equation at the $k$ th node for the specified circuital graph, in which generic admittances are considered, the circuit $k$ th module of the analog is obtained as specified in Fig. 5, if the inductance $L$ and the capacitance $C$ fulfill the following condition:

$$
L C=\frac{\alpha^{4}}{\varpi^{2}} \delta^{4}=\frac{\rho}{K_{M}}(\delta l)^{4} .
$$

\footnotetext{
${ }^{3}$ Characteristic potentials $V_{0}$ will be introduced in the next section while discussing the governing equations for the PEM beam and its electric analogs.
} 
2.2.1.1. Synthesis of analog circuits for mechanical constraints. Since we are interested in the control and damping of vibrations in beam frames, it is necessary to understand how to electrically synthesize, in the analog circuit, the boundary conditions equivalent to the mechanical ones. Indeed the equivalence of the boundary conditions, together with the analogy of the governing differential equations between the electrical and the mechanical subsystems, guarantees a complete electro-mechanical modal matching.

Simple consideration, based on the finite difference rule, allow such a synthesis for the generic mechanical constraint: as an example in Figs. 7 and 8 the analog of a clamping and hinge device are provided.

\subsubsection{Synthesis of the analog circuit based on the mobility matrix method}

Let us consider the following normal system associated to (7):

$$
\tilde{\mathbf{v}}^{\prime}(x, s)=\mathbf{A}(s) \tilde{\mathbf{v}}(x, s),
$$

where

$$
\tilde{\mathbf{v}}^{\prime}(x, s)=\left[\begin{array}{c}
\tilde{v}(x, s) \\
\tilde{v}^{\prime}(x, s) \\
\tilde{M}(x, s) \\
\tilde{T}(x, s)
\end{array}\right], \quad \mathbf{A}(s)=\left[\begin{array}{cccc}
0 & 1 & 0 & 0 \\
0 & 0 & s & 0 \\
0 & 0 & 0 & -1 \\
\alpha^{4} s^{2} & 0 & 0 & 0
\end{array}\right] .
$$

Hence the column vector $\tilde{\mathbf{v}}$ evaluated at $(k+1)$ th sampling node can be related to its evaluation at the $k$ th node by:

$$
\tilde{\mathbf{v}}_{k+1}(s)=\mathrm{e}^{\delta \mathbf{A}(s)} \tilde{\mathbf{v}}_{k}(s) .
$$

Regarding the beam element between $k$ th and the $(k+1)$ th mesh nodes as the mechanical black box "beam elements" the exponential matrix $\mathrm{e}^{\delta \mathbf{A}(s)}$ is the transmission matrix of the beam element (Molloy, 1958), since it relates the "input" of the black box $\tilde{\mathbf{v}}_{k}(s)$ to its "output" $\tilde{\mathbf{v}}_{k+1}(s)$.

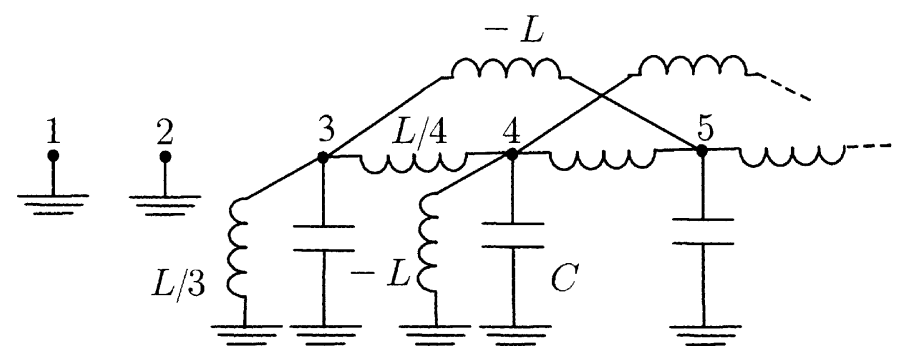

Fig. 7. Circuital analog for the clamping device.

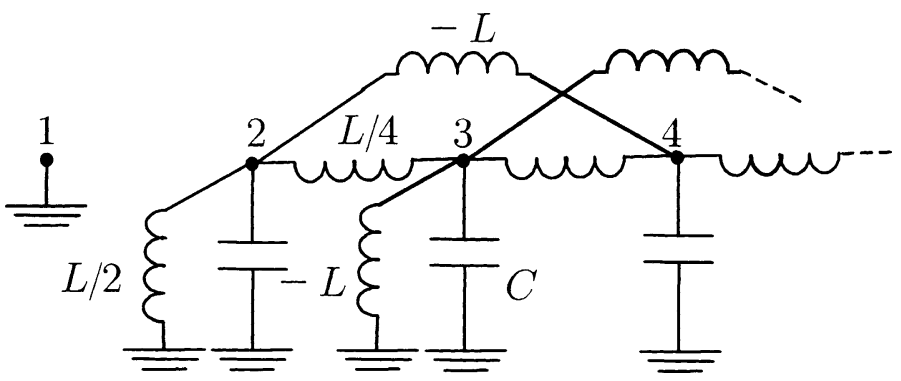

Fig. 8. Circuital analog for the hinge. 
Let us explicitly remark that (14) represents exactly the relation between the values of $\tilde{\mathbf{v}}(x, s)$ at two adjacent sampling nodes without introducing any approximation with respect to the continuum model.

For our purposes it is fruitful to consider the mobility matrix $\mathbf{B}(\delta, s)$ of the same $(k, k+1)$ beam element, obtained from the (14) as the matrix relating the kinematical descriptors

$$
\left[\begin{array}{llll}
\tilde{v}_{k} & \tilde{v}_{k}^{\prime} & \tilde{v}_{k+1} & \tilde{v}_{k+1}^{\prime}
\end{array}\right]^{\mathrm{T}}
$$

to the contact actions

$$
\left[\begin{array}{llll}
-\tilde{T}_{k} & -\tilde{M}_{k} & \tilde{T}_{k+1} & \tilde{M}_{k+1}
\end{array}\right]^{\mathrm{T}}
$$

exerted on the beam element (see Molloy, 1958; Newcomb, 1966).

By means of this representation the power expended by the outer world on the beam element is equal to the inner product between the kinematical and contact actions vectors.

The generic entry of the mobility matrix has the following form:

$$
B_{i j}(\delta, s)=\frac{h_{i j}(\delta, s)}{-1+\cosh \left(\mathrm{e}^{\mathrm{i}(\pi / 4)} \alpha \delta \sqrt{s}\right) \cos \left(\mathrm{e}^{\mathrm{i}(\pi / 4)} \alpha \delta \sqrt{s}\right)}, \quad i, j=1, \ldots, 4,
$$

where $h_{i j}(\delta, s)$ is a holomorphic function of the beam element size $\delta$ and the Laplace variable $s$, thus $B_{i j}$ is a meromorphic function.

Since $B_{i j}$ has an infinite number of poles, it is not possible to simulate exactly the beam element by a finite lumped circuit; hence a suitably truncated Laurent expansion of the mobility matrix with respect of the size $\delta$ in the neighborhood of the pole at zero is performed. The ring of convergence of the expansion is determined by the absolute value of the first non-zero root of the denominator in (15), that is:

$$
\delta_{\max } \simeq \frac{4.73004}{\alpha \sqrt{\omega}}
$$

where $\omega=\operatorname{Im}[s]$.

Condition (16) establishes an upper bound for the mesh size $\delta$ once the highest vibration frequency of the beam to be controlled is fixed. Truncating the Laurent expansion at the third power of $\delta$ we obtain for $\mathbf{B}$ a Foster's canonic form (Newcomb, 1966) relative to a completely passive finite lumped circuit. Therefore the following approximate mobility matrix is found:

$$
\mathbf{B}(\delta, s) \simeq \frac{1}{s} \mathbf{K}_{0}(\delta)+s \mathbf{K}_{\infty}(\delta),
$$

where both the residue matrices are symmetric and positive semi-definite.

The analog circuit for the beam element can be designed as a completely passive four port electric network as depicted in Fig. 9, the dimensionless impedance matrix of which is equal to the approximate mobility matrix $\mathbf{B}(\delta, s)$, given by the RHS of (17). Indeed because of the listed properties for the residue matrices the analog network can be synthesized as the series connection of two networks, see Newcomb (1966), the former being realized by two uncoupled capacitors terminating a multiport transformer and the latter by four uncoupled inductors terminating a second multiport transformer.

The values of the uncoupled impedances are determined by the eigenvalues of the residue matrices in (17), while the eigenvectors of these matrices characterize the turns ratio matrices of the two ideal transformers.

Cumbersome but simple calculations show that the needed inductances $L_{i}(i=1, \ldots, 4)$ and capacitances $C_{1}, C_{2}$, together with the turns ratio matrices $T_{0}, T_{\infty}$ are given by: 


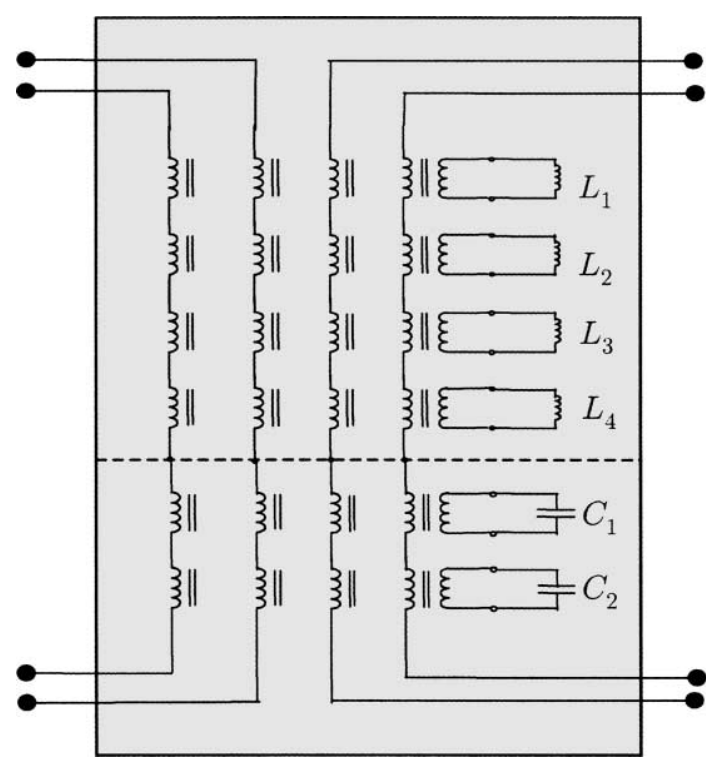

Fig. 9. Analog circuit for the beam element.

$$
\begin{aligned}
& \mathbf{T}_{0}=\frac{\kappa_{0}}{\sqrt{2}}\left[\begin{array}{cccc}
1 & 0 & 1 & 0 \\
-\frac{1}{2} \delta & 1 & \frac{1}{2} \delta & 1
\end{array}\right], \\
& \mathbf{T}_{\infty}=\frac{\kappa_{\infty}}{\sqrt{2}}\left[\begin{array}{cccc}
\frac{1}{6} \delta & -1 & \frac{1}{6} \delta & 1 \\
1 & \frac{1}{6} \delta & 1 & -\frac{1}{6} \delta \\
-\frac{3}{34} \delta & 1 & \frac{3}{34} \delta & 1 \\
1 & \frac{3}{34} \delta & -1 & \frac{3}{34} \delta
\end{array}\right], \\
& \frac{C_{1}}{C_{2}}=\frac{12}{\delta^{2}}, \quad C_{1} L_{2}=\frac{1}{720} \varsigma^{2}, \quad C_{2} L_{1}=\frac{1}{48} \varsigma^{2}, \quad C_{2} L_{3}=\frac{17}{4680} \varsigma^{2}, \quad C_{1} L_{4}=\frac{1}{4080} \varsigma^{2}, \\
& \varsigma^{2}=\left(\frac{\kappa_{0}}{\kappa_{\infty}}\right)^{2} \frac{\alpha^{4}}{\pi^{2}} \delta^{4}=\left(\frac{\kappa_{0}}{\kappa_{\infty}}\right)^{2} \frac{\rho}{K_{M}}(\delta l)^{4},
\end{aligned}
$$

where the coefficients $\kappa_{0}$ and $\kappa_{\infty}$ are free for contingent design needs.

The applied synthesis technique allows for an immediate mechanical interpretation of the port voltages and currents: the port voltages represent the velocity deflection and angular velocity at the two ends of the beam element, while the port currents represent the contact actions (bending moment and shear force) exerted by the outer world on the beam element. As a consequence, the circuit analog for the entire beam is easily obtained by means of a cascade connection of the four port networks equivalent to the beam elements (see Fig. 10).

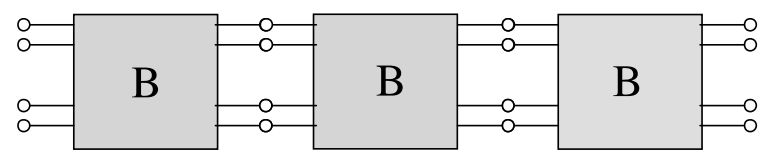

Fig. 10. Cascade connection of the beam-elements analog circuits. 
Furthermore the mechanical boundary conditions are naturally imposed properly terminating the ports of the boundary modules. For instance a clamped end is simulated short circuiting both of the ports of the edge module; this connection corresponds to set the boundary deflection velocity and angular velocity to zero.

Let us explicitly remark that the circuital beam element synthesized can be realized in a completely passive way and that considerably low performances are requested to all the electric components.

\section{PEM beam}

A PEM beam is obtained uniformly distributing a set of bending piezoelectric actuators on a beam and using them as two terminal active networks in a beam analog circuit.

\subsection{Distributed piezoelectric effect}

A bending actuator (shown in Fig. 11) can be regarded as a lossless lumped transducer having one mechanical and one electrical terminal. The mechanical state is characterized by the bending deformation $\Delta \theta_{P}$ and the exerted couple $M_{P}$ while its electrical state is characterized by the potential drop $V_{P}$ between its plates and the stored capacitive charge $Q_{P}$.

Usually a piezoelectric actuator operates in linear quasi-static regimes. Therefore its behavior can be modelled by the following linear constitutive equation

$$
\left[\begin{array}{l}
M_{P} \\
Q_{P}
\end{array}\right]=\left[\begin{array}{cc}
k_{\mathrm{mm}} & k_{\mathrm{me}} \\
-k_{\mathrm{me}} & k_{\mathrm{ee}}
\end{array}\right]\left[\begin{array}{c}
\Delta \theta_{P} \\
V_{P}
\end{array}\right],
$$

where $k_{\mathrm{mm}}$ is the actuator bending stiffness, $k_{\mathrm{ee}}$ its capacitance and $k_{\mathrm{me}}$ its electro-mechanical coupling coefficient.

From an electric point of view, the actuator can be regarded as a capacitor in parallel connection with a current source as shown in Fig. 12: the imposed current value depends on the bending deformation speed. This circumstance suggests to use the actuators in the analog circuit whenever a capacitor is needed.

Thus, the first circuital scheme for the PEM beam is realized simply connecting one electric terminal of each actuator to the ground node and the second one to every $k$ th node according to Fig. 13. On the other hand it is easy to prove that a convenient choice for an efficient energy transfer in the second scheme (see Fig. 14) is obtained replacing the sole capacitance $C_{1}$ by the piezoelectric actuator. Indeed it can be proven that a current source parallel to the capacitor $C_{2}$ provides a comparatively negligible energy transfer.

The piezoelectric current sources depending on the bending deformation speed (see Porfiri, 2000; Alessandroni, 2000), provide a non-homogeneous term in the partial differential equation governing the voltage evolution in the electric network:

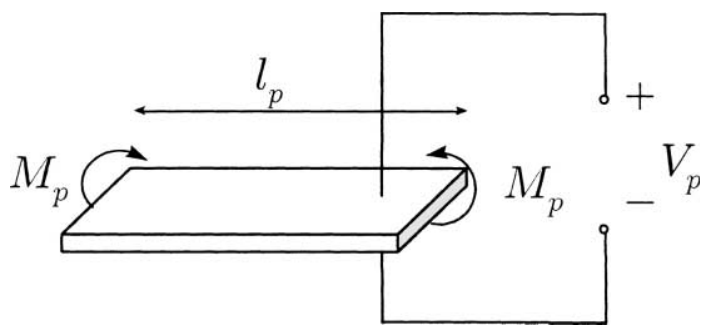

Fig. 11. Bending PZT actuator. 


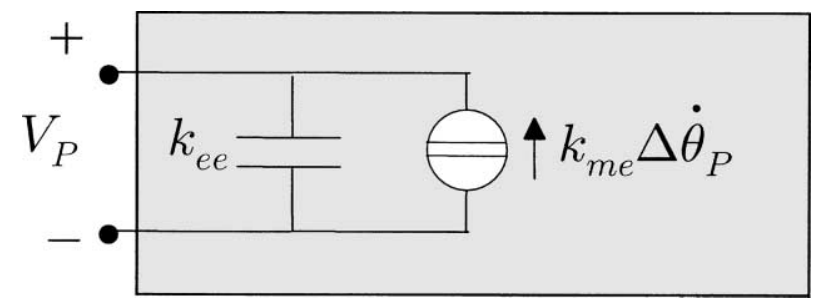

Fig. 12. Electric scheme of a bending PZT actuator.

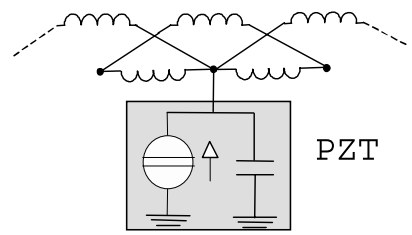

Fig. 13. PZT insertion in the "finite differences" analog circuit.

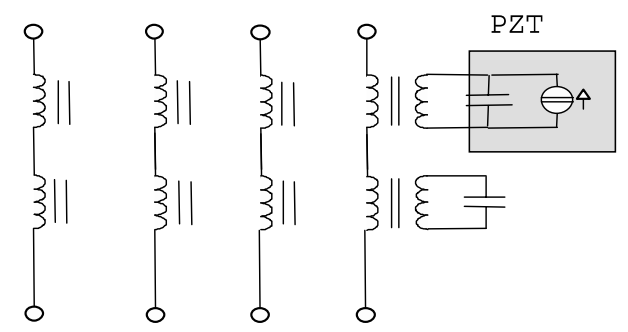

Fig. 14. PZT insertion in the "mobility matrix" analog circuit.

$$
V^{\mathrm{IV}}+\alpha^{4} \ddot{V}-\beta_{\mathrm{em}}^{2} \dot{v}^{\prime \prime}=0 .
$$

Eq. (22) is the result of an homogenization procedure performed for both the mentioned analog circuits. The e-m coupling coefficient are given by Table 1, where $\eta$ is the piezoelectric covering factor, defined as the percentage of beam length covered by the piezo-layer. From a mechanical point of view, the actuators modify the constitutive equation for the bending moment (21), introducing an additional mechanical bending stiffness and a surplus of bending moment linearly depending on the piezoelectric voltage. As it is reasonable to consider the bending stiffness and mass per unit length of the piezo-layer negligible with

Table 1

Electro-mechanical coupling coefficients

\begin{tabular}{ll}
\hline Circuit in Fig. 14 & Circuit in Fig. 14 \\
\hline$\beta_{\mathrm{em}}^{2}=-\frac{\kappa_{0}}{\sqrt{2}} \frac{k_{\mathrm{me}} l^{3} \delta \rho \varpi^{2} \eta}{K_{M} k_{\mathrm{ee}}} \frac{h}{V_{0}}$ & $\beta_{\mathrm{em}}^{2}=\frac{k_{\mathrm{me}} l^{3} \delta \rho \varpi^{2} \eta}{K_{M} k_{\mathrm{ee}}} \frac{h}{V_{0}}$ \\
$\beta_{\mathrm{me}}^{2}=-\frac{\sqrt{2}}{\kappa_{0}} \frac{k_{\mathrm{me}} l^{2} \eta}{K_{M}} \frac{V_{0}}{h}$ & $\beta_{\mathrm{me}}^{2}=\frac{k_{\mathrm{me}} l^{2} \eta}{K_{M}} \frac{V_{0}}{h}$ \\
\hline
\end{tabular}




$$
\begin{array}{ll}
V_{0}=\frac{h \varpi \kappa_{0}}{\sqrt{2}} \sqrt{\frac{\rho \delta l}{k_{\mathrm{ee}}}} \beta & V_{0}=h \varpi \sqrt{\frac{\rho \delta l}{k_{\mathrm{ee}}}} \\
\beta^{2}=-\frac{k_{\mathrm{me}} l^{2} \varpi}{K_{M}} \eta \sqrt{\frac{\rho \delta l}{k_{\mathrm{ee}}}} & \beta^{2}=\frac{k_{\mathrm{me}} l^{2} \varpi}{K_{M}} \eta \sqrt{\frac{\rho \delta l}{k_{\mathrm{ee}}}}
\end{array}
$$

respect of the proper bending stiffness and mass per unit length of the beam, the following second coupled electro-mechanical equation for the PEM beam is obtained

$$
v^{\mathrm{IV}}+\alpha^{4} \ddot{v}+\beta_{\mathrm{me}}^{2} \dot{V}^{\prime \prime}=0,
$$

where the $\mathrm{m}-\mathrm{e}$ coupling coefficient is given by Table 1 . We can choose the characteristic potential $V_{0} \mathrm{im}$ posing that $\beta_{\mathrm{me}}^{2}=\beta_{\mathrm{em}}^{2}$, so to obtain the expressions for the coupling coefficient $\beta$ given in Table 2 .

In conclusion the evolution equations for a conservative PEM beam are: ${ }^{4}$

$$
\left[\begin{array} { l } 
{ v ^ { \mathrm { IV } } + \alpha ^ { 4 } \ddot { v } + \beta ^ { 2 } \dot { V } ^ { \prime \prime } = 0 , } \\
{ V ^ { \mathrm { IV } } + \alpha ^ { 4 } \ddot { V } - \beta ^ { 2 } \dot { v } ^ { \prime \prime } = 0 , }
\end{array} \left[\begin{array}{l}
\alpha^{4}=\varpi^{2} l^{4} \frac{\rho}{K_{M}}, \\
\beta^{2}= \pm \frac{l^{2} \varpi g_{\mathrm{me}}}{K_{M}} \eta \sqrt{\frac{\delta l \rho}{k_{\mathrm{ee}}}} .
\end{array}\right.\right.
$$

The evolution equations expressed in terms of the kinematical descriptors $u$ and $\psi$ (dimensionless transverse displacement and flux linkage) become:

$$
\left[\begin{array}{l}
u^{\mathrm{IV}}+\alpha^{4} \ddot{u}+\beta^{2} \dot{\psi}^{\prime \prime}=0 \\
\psi^{\mathrm{IV}}+\alpha^{4} \ddot{\psi}-\beta^{2} \dot{u}^{\prime \prime}=0
\end{array}\right.
$$

where $\dot{\psi}=V$.

\subsection{PEM beam performances analysis}

Two different approaches for the analysis of the PEM beam model (25) are addressed here. The former considers an electro-mechanical wave freely propagating in an PEM beam, while the latter contemplates a modal evolution for a constrained PEM beam.

\subsubsection{Propagation properties}

Let us consider an electro-mechanical wave of wavelength $\lambda$ and angular frequency $\omega$ freely propagating in a PEM beam:

$$
\left[\begin{array}{l}
u \\
\psi
\end{array}\right](x, t)=\left[\begin{array}{l}
A \\
B
\end{array}\right] \exp (j((2 \pi / \lambda) x-\omega t)),
$$

where $A$ and $B$ are respectively the mechanical and electric complex wave amplitudes. The dispersive relations for the conservative PEM beam can be decomposed in the following form:

$$
\omega_{+,-}(\lambda)=\left(\omega_{\mathrm{c}}(\lambda) \pm \omega_{\mathrm{e}}(\lambda)\right)
$$

\footnotetext{
${ }^{4}$ In order to have the same algebraic signs for both the synthesized conservative PEM beam, it is sufficient to choose a negative $\kappa_{0}$.
} 


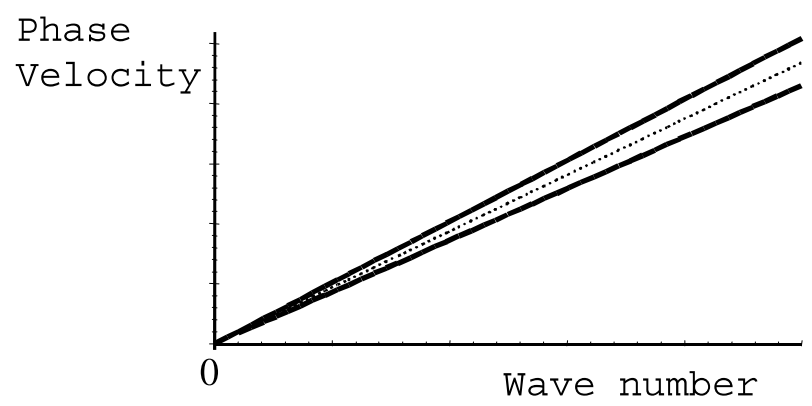

Fig. 15. Dispersion relation in a conservative PEM beam.

where

$$
\begin{aligned}
& \omega_{\mathrm{c}}(\lambda)=\sqrt{1+\left(\frac{\beta^{2}}{2 \alpha^{2}}\right)^{2}} \omega_{\mathrm{o}}(\lambda), \\
& \omega_{\mathrm{e}}(\lambda)=\frac{\beta^{2}}{2 \alpha^{2}} \omega_{\mathrm{o}}(\lambda), \\
& \omega_{\mathrm{o}}(\lambda)=\left(\frac{\lambda}{\alpha}\right)^{2} .
\end{aligned}
$$

The term $\omega_{0}$ represents the frequency of the two waves propagating independently in the uncoupled electrical and mechanical supports. The terms $\omega_{\mathrm{e}}$ and $\omega_{\mathrm{c}}$ can be regarded as the envelope and the carrier frequencies ${ }^{5}$ of the electro-mechanical signal:

$$
\begin{aligned}
& \omega_{\mathrm{c}}=\frac{\omega_{+}+\omega_{-}}{2}, \\
& \omega_{\mathrm{e}}=\frac{\omega_{+}-\omega_{-}}{2} .
\end{aligned}
$$

Hence the coupling effect generates a sinusoidal modulation $\omega_{\mathrm{e}}$ on the carrier $\omega_{\mathrm{c}}$, due to the energy transfer between the electrical and the mechanical wave components.

In Fig. 15 the phase speed $v_{p}$ versus the wave number ${ }^{6} k$ for the uncoupled (dash line) case and the coupled (solid lines) one is drawn showing the splitting of the solution around the uncoupled curve given by the coupling.

Furthermore, the relation between the electric and mechanical wave amplitudes

$$
A(\omega)=\mathrm{i} B(\omega),
$$

states that the relative intensity of the electrical and mechanical wave components is always the same independently of the frequency, i.e. every electro-mechanical plane wave propagating in the PEM beam contains an equal amount of electrical and mechanical energy. Moreover Eq. (29) states that, as the wave electric component is in quadrature with respect to the mechanical one, the energy of the system completely flows back and forth from one form to the other.

\footnotetext{
${ }^{5}$ The concepts of carrier and envelope frequencies directly derive from the theory of signals, dealing, for instance, with amplitudemodulation (AM) broadcasting communications.

${ }^{6}$ The phase speed is defined as the ratio between the frequency $\omega_{+,-}$and the wave number $k=2 \pi / \lambda$.
} 
Hence, as expected if the PEM beam is designed exploiting the circuital analogs an optimal coupling, is really obtained, which is furthermore completely independent of the frequency of the wave freely propagating in it.

\subsubsection{Coupled modal analysis}

In this subsection a modal analysis for a simply supported PEM beam (shown in Fig. 16), based upon the Galerkin method, is performed.

The set of basis functions chosen to represent the deflection $u(x, t)$ and the flux linkage $\psi(x, t)$ of the PEM beam is constituted by the eigenfunctions $\varphi_{m}(x)$ of the spatial differential operator determining the modal forms for both the uncoupled motions:

$$
\varphi_{m}(x)=\sqrt{2} \sin \left(\frac{2 \pi}{\lambda_{m}} x\right), \quad \lambda_{m}=\frac{2}{m}, \quad m=1,2, \ldots
$$

Consequently a Fourier expansion for the solution of (25) is considered:

$$
\left[\begin{array}{l}
u(x, t) \\
\psi(x, t)
\end{array}\right]=\sum_{m=1}^{\infty}\left[\begin{array}{l}
p_{m}(t) \\
q_{m}(t)
\end{array}\right] \varphi_{m}(x)
$$

where $p_{m}(t)$ and $q_{m}(t)$ are respectively the mechanical and electrical Fourier coefficients of the expansion. With simple calculation the following state space system for the ODEs governing the time evolution of a generic pair of electrical and mechanical Fourier coefficients is obtained

$$
\frac{\mathrm{d}}{\mathrm{d} t}\left[\begin{array}{c}
p_{m}(t) \\
q_{m}(t) \\
\dot{p}_{m}(t) \\
\dot{q}_{m}(t)
\end{array}\right]=\left[\begin{array}{cccc}
0 & 0 & 1 & 0 \\
0 & 0 & 0 & 1 \\
-\left(\frac{m \pi}{\alpha}\right)^{4} & 0 & 0 & \frac{\beta^{2}(m \pi)^{2}}{\alpha^{4}} \\
0 & -\left(\frac{m \pi}{\alpha}\right)^{4} & -\frac{\beta^{2}(m \pi)^{2}}{\alpha^{4}} & 0
\end{array}\right]\left[\begin{array}{c}
p_{m}(t) \\
q_{m}(t) \\
\dot{p}_{m}(t) \\
\dot{q}_{m}(t)
\end{array}\right]
$$

Let us explicitly remark that the chosen boundary conditions and basis functions lead to a set of uncoupled evolution problems for each electro-mechanical mode, hence it is possible to easily derive the analytical solution for the Fourier coefficients.

The set of natural frequencies of the PEM beam is determined by the characteristic polynomials $D_{m}(s)=\operatorname{det}\left(\mathbf{A}_{m}-s \mathbf{I}\right)$ of the evolution matrices $\mathbf{A}_{m}$, in Eq. (30):

$$
D_{m}(s)=s^{4}+2\left[\left(\omega_{\mathrm{c}}^{m}\right)^{2}+\left(\omega_{\mathrm{e}}^{m}\right)^{2}\right] s^{2}+\left[\left(\omega_{\mathrm{c}}^{m}\right)^{2}-\left(\omega_{\mathrm{e}}^{m}\right)^{2}\right]^{2}=0,
$$

where $\omega_{\mathrm{c}}^{m}$ and $\omega_{\mathrm{e}}^{m}$ coincide with the envelope and carrier frequencies in (27) evaluated at the modal wavelengths:

$$
\begin{aligned}
& \omega_{\mathrm{c}}^{m}=\left.\omega_{\mathrm{c}}^{m}(\lambda)\right|_{2 / m}, \\
& \omega_{\mathrm{e}}^{m}=\left.\omega_{\mathrm{e}}^{m}(\lambda)\right|_{2 / m} .
\end{aligned}
$$

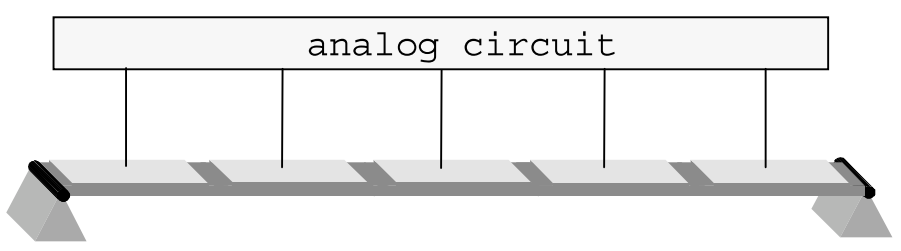

Fig. 16. Simply supported PEM beam. 
Considering the following initial conditions for (25)

$$
\begin{aligned}
& \left.u(x, t)\right|_{t=0}=\sum_{m=1}^{\infty} p_{m}^{0} \varphi_{m}(x), \\
& \left.\psi(x, t)\right|_{t=0}=0 \\
& \left.\dot{u}(x, t)\right|_{t=0}=0, \\
& \left.\dot{\psi}(x, t)\right|_{t=0}=0
\end{aligned}
$$

the following time evolution for the coefficients of $p_{m}, q_{m}$ is derived:

$$
\left[\begin{array}{l}
p_{m}(t)=p_{m}^{0} \cos \left(\omega_{m}^{\mathrm{e}} t\right) \cos \left(\omega_{m}^{\mathrm{c}} t\right)+p_{m}^{0} \frac{\omega_{m}^{\mathrm{c}}}{\omega_{m}^{m}} \sin \left(\omega_{m}^{\mathrm{e}} t\right) \sin \left(\omega_{m}^{\mathrm{c}} t\right), \\
q_{m}(t)=p_{m}^{0} \sin \left(\omega_{m}^{\mathrm{e}} t\right) \cos \left(\omega_{m}^{\mathrm{c}} t\right)-p_{m}^{0} \frac{\omega_{m}^{m}}{\omega_{m}^{\mathrm{e}}} \cos \left(\omega_{m}^{\mathrm{e}} t\right) \sin \left(\omega_{m}^{\mathrm{c}} t\right),
\end{array}\right.
$$

which again (see (29)) exhibits how the evolution for the electro-mechanical mode is composed by two modulated signals in quadrature. Figs. 17 and 18 again show how the energy goes back and forth from the mechanical to the electric form: they are obtained for an aluminum PEM beam the constitutive piezoelectro-mechanical coefficient of which are specified in Table 3.

The used piezoelectric transducer are piezoceramic patches (T110-H4E-602 (PSI-5H4)) made of lead zirconate titanium (PZT) produced by Piezo Systems (Piezo Systems). Remark that once the mechanical
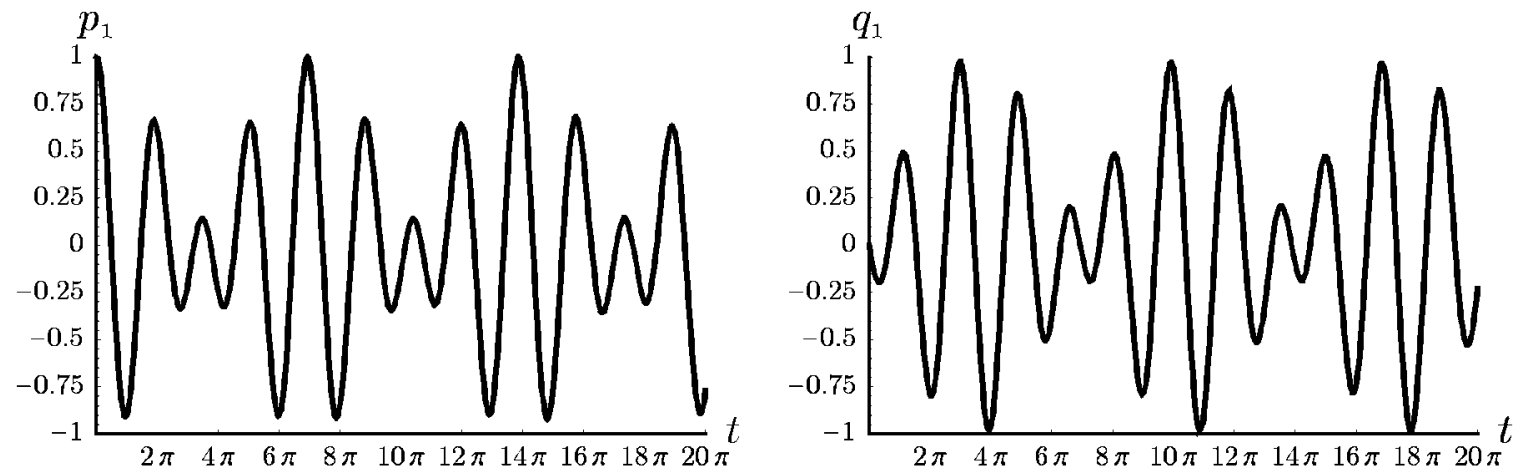

Fig. 17. Time evolution of the first Fourier electrical and mechanical coefficients.

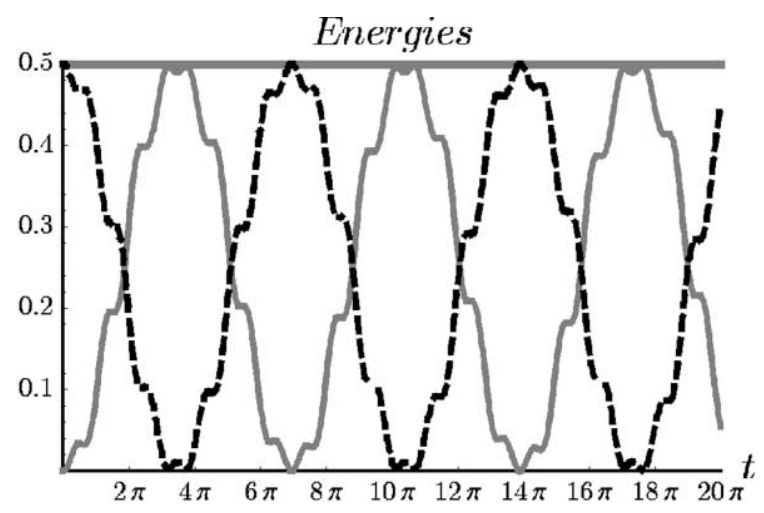

Fig. 18. Mechanical (dash) and electrical (solid) energy time evolutions. 


\begin{tabular}{ll} 
Beam length & $l=1 \mathrm{~m}$ \\
Sides of rectangular section & $4 \mathrm{~mm} \times 5 \mathrm{~cm}$ \\
Mass density per unit length & $\rho=.54 \mathrm{~kg} / \mathrm{m}$ \\
Bending stiffness & $K_{M}=18.67 \mathrm{~N} \mathrm{~m}^{2}$ \\
PZT capacitance & $k_{\text {ee }}=1.02 \times 10^{-6} \mathrm{~F}$ \\
PZT coupling coefficient & $k_{\mathrm{me}}=3.97 \times 10^{-3}(\mathrm{~N} / \mathrm{V}) \mathrm{m}=\mathrm{C}$ \\
PZT covering factor & $\eta=1$ \\
Number of actuators & 10 \\
Characteristic frequency & $\varpi=\left(\pi^{2} / l^{2}\right) \sqrt{K_{M} / \rho}=58 \mathrm{rad} / \mathrm{s}$ \\
Characteristic tension & $V_{0}=53.4 \mathrm{~V}$ \\
\hline
\end{tabular}

Table 4

Values of the electrical elements in the analog circuits

\begin{tabular}{ll}
\hline Circuit in Fig. 5 & Circuit in Fig. 9 \\
\hline Negative inductance: $2.83 \mathrm{H}$ & Maximum inductance: $70 \mathrm{mH}$ \\
Positive inductance: $707 \mathrm{mH}$ & Turns ratio (magnitude order): $1 \div 100$ \\
\hline
\end{tabular}

parameters are fixed the electrical ones are given by Eqs. (18) and (20) in the case of the circuit in Fig. 5 or by Eq. (10) in the case of the circuit in Fig. 9, as presented in Table 4 for the considered PEM beam.

\subsubsection{Electro-mechanical damping}

Once it is proven that conservative PEM beams assure an efficient electro-mechanical coupling, it is reasonable to investigate the possibility of damping mechanical vibrations via dissipative elements to be inserted in the electric circuit. This can be done adding to the circuit sketched in Fig. 14 a resistor in parallel connection with each actuator (see Fig. 19).

Going through calculations similar to those detailed in Section 2, it is easy to derive the governing equation for the so defined "electrically dissipative PEM beam":

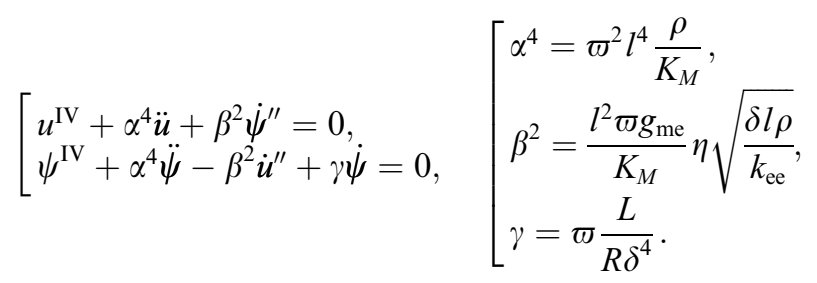

The dissipative electric circuit so obtained is the electric analog for a beam connected to a bed of mechanical dampers as in Fig. 20, or equivalently for a beam embedded in a viscous fluid.

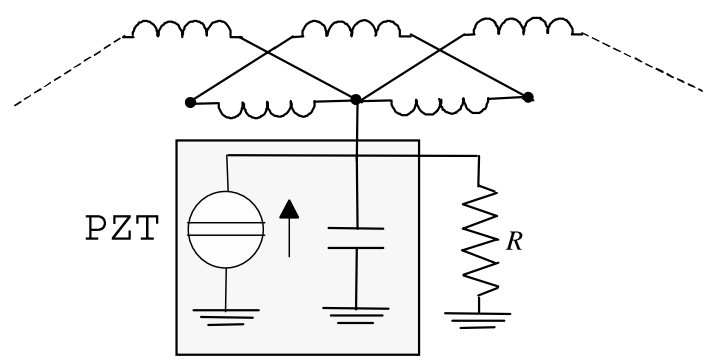

Fig. 19. Dissipative circuital module in the "finite differences" analog circuit. 


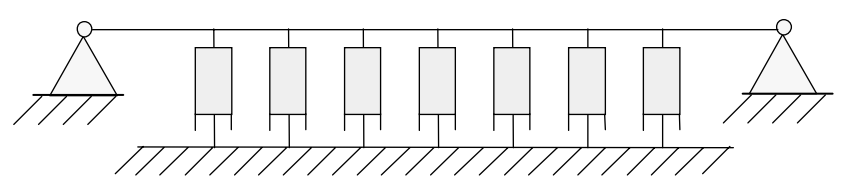

Fig. 20. Beam over a bed of dampers.

The analysis of the dissipative system is performed as for the conservative PEM beam: indeed the evolution matrix in the (30) must be slightly modified simply including an extra non-zero diagonal term:

$$
\overline{\mathrm{d} t}\left[\begin{array}{c}
p_{m}(t) \\
q_{m}(t) \\
\dot{p}_{m}(t) \\
\dot{q}_{m}(t)
\end{array}\right]=\mathbf{B}_{m}\left[\begin{array}{c}
p_{m}(t) \\
q_{m}(t) \\
\dot{p}_{m}(t) \\
\dot{q}_{m}(t)
\end{array}\right],
$$

with

$$
\mathbf{B}_{m}=\left[\begin{array}{cccc}
0 & 0 & 1 & 0 \\
0 & 0 & 0 & 1 \\
-\left(\frac{m \pi}{\alpha}\right)^{4} & 0 & 0 & \frac{\beta^{2}(m \pi)^{2}}{\alpha^{4}} \\
0 & -\left(\frac{m \pi}{\alpha}\right)^{4} & -\frac{\beta^{2}(m \pi)^{2}}{\alpha^{4}} & -\frac{\gamma}{\alpha^{4}}
\end{array}\right] .
$$

We remark that:

1. the evolution matrix $\mathbf{B}_{m}$ in (35) is obtained from $\mathbf{A}_{m}$ replacing the vanishing coefficient $\mathbf{A}_{m_{44}}$ with $-\gamma / \alpha^{4}$.

2. It is possible to represent the characteristic polynomial $P_{m}(s)$ of $\mathbf{B}_{m}$ as the sum of the "non-dissipative polynomial" $D_{m}(s)$ defined in (31) and a term linearly dependent on the dissipation coefficient $\gamma$ :

$$
\begin{aligned}
& P_{m}(s)=D_{m}(s)+\frac{\gamma}{\alpha^{4}} N_{m}(s), \\
& N_{m}(s)=s\left[s^{2}+\left(\omega_{\mathrm{o}}^{m}\right)^{2}\right] .
\end{aligned}
$$

This representation of the characteristic polynomial suggests to consider $P_{m}(s)$ as the denominator of the transfer function of the closed-loop system in Fig. 21, where the dissipation coefficient $\gamma$ plays the role of a feedback amplifier, the aim of which is to displace the poles of the non-dissipative system from the imaginary axis.

Following the methods widely applied in the theory of control, it is useful to analyze the location of the poles of the closed-loop transfer function with respect to the parameter $\gamma$ by means of the root locus technique. In Fig. 22 the root locus of the characteristic polynomial $P_{m}(s)$ is represented as a set of curves parametrized by $\gamma$ varying in $\mathbb{R}^{+}$.

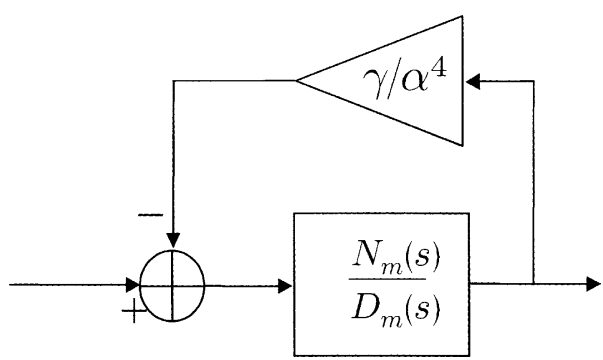

Fig. 21. Closed-loop system. 


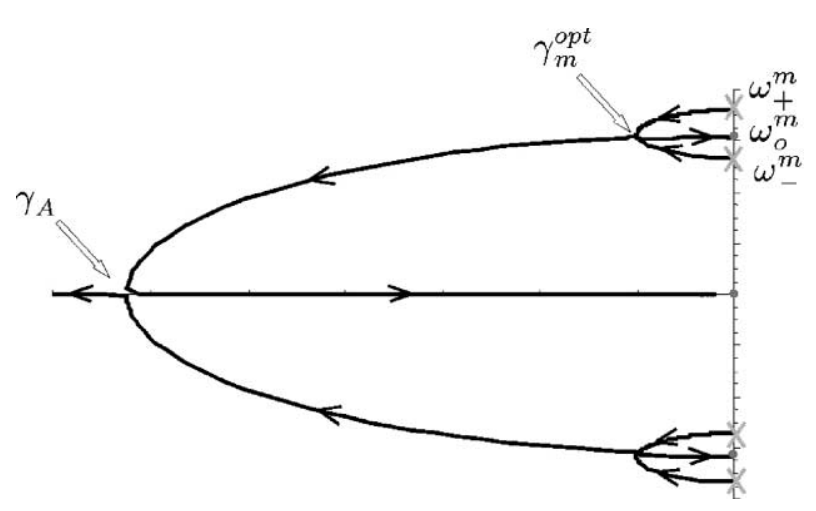

Fig. 22. Root locus of the characteristic polynomial $P_{m}(s)$ varying the parameter $\gamma$.

At $\gamma=0$ (starting points of the root locus) the resistances tend to infinity, so that the roots lie on the imaginary axis and represent the natural frequencies of the $m$ th mode conservative PEM beam.

Conversely, as $\gamma$ tends to infinity (end points of the root locus) the resistances vanish, short circuiting the actuators to ground. Thus the electric circuit is not apt any more to support the electric wave propagation and the coupling effect is definitively lost.

Furthermore, the end points of the root locus, are formed by a couple of purely imaginary conjugate roots $\pm j \omega_{\mathrm{o}}^{m}$ (describing the evolution of the free vibrating beam) and other two purely real valued roots which tend respectively to zero and to $-\infty$.

In fact there exists a threshold value $\gamma_{A}$ such that when $\gamma>\gamma_{A}$ two roots for $P_{m}$ are purely real. This last circumstance states mathematically that when the shunting resistance is small enough the electric circuit loses its propagative properties, the PZT actuators are practically short circuited and PEM beams reduce to standard beams.

Finally it can be proven that for

$$
\gamma=\gamma_{m}^{\mathrm{opt}}=2 \beta^{2}(m \pi)^{2}
$$

the real parts of the four roots of $P_{m}$ coincides and equal $-\left(\beta^{2} / \alpha^{4}\right)(m \pi)^{2}$. The corresponding value for the shunt resistance will be assumed to be the optimal value for an efficient and quick mechanical damping. This assumption is well grounded, as:

1. when $\gamma=\gamma_{m}^{\text {opt }}$ the damping ratios of the considered electro-mechanical modes for the dissipative PEM beam have a common value $\zeta_{m}^{\text {opt }}$,

2. when $\gamma \neq \gamma_{m}^{\text {opt }}$ there exist modes the damping ratio of which is smaller than $\zeta_{m}^{\text {opt }}$.

From a mechanical point of view the existence of the optimal value $\gamma_{m}^{\text {opt }}$ can be interpreted as follows:

1. Too high-shunt resistances (i.e. low values for $\gamma$ ) allow for a good electro-mechanical coupling, but provide a weak dissipation. Thus the energy still goes back and forth from one form to the other and the time evolution for the mechanical Fourier coefficient $p_{m}$ (considering, as before, a sole initial deflection) still presents a pseudo-periodic decreasing envelope. In Fig. 23 it is presented (for the PEM beam in Table 3) the high-shunt-resistance time-evolution of the first mechanical Fourier coefficient: this figure and all the following ones are relative to initial data of type specified in 33 , where only $p_{1}^{0}$ is different from zero. 


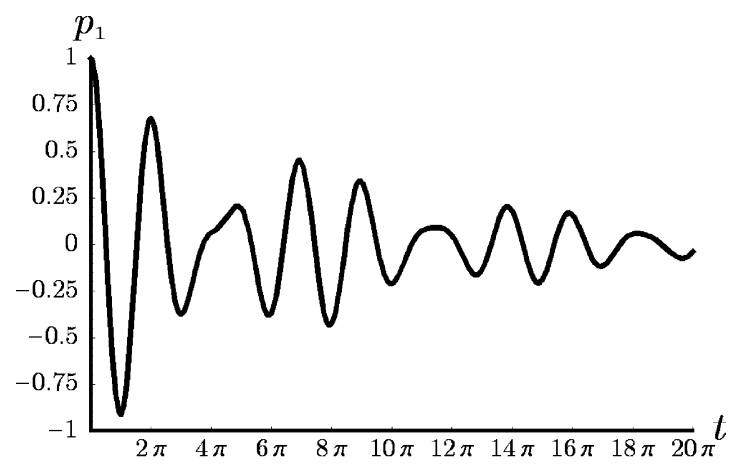

Fig. 23. Too high-shunt resistance time evolution.

2. On the other hand, too low-value resistances (i.e. high values of $\gamma$ ) interdict an efficient coupling between the two systems slowing down the flowing of energy from one form to the other. Consequently, because of the lack of coupling, the evolution of $p_{m}$ does not present any more an oscillating envelope, while a decreasing exponential slope related to the real part of the nearest pole to the imaginary axes is evident. In Fig. 24 it is presented (for the PEM beam in Table 3) the low-shunt-resistance time-evolution of the first mechanical Fourier coefficient.

The previous considerations substantiate the optimum criterion we have introduced: a reasonable optimal damping for the mechanical vibration via electric dissipation can be obtained imposing that the absolute value for the real part of the nearest pole to the imaginary axes is maximum, which corresponds to request to the lowest damping ratio of the poles to be maximum as a function of $\gamma$. This is the "pole placement" criterion (see e.g. Hagood and von Flotow, 1991). This criterion does not explicitly refer to the electrical dissipation of the mechanical energy. However both the analysis of the eigenvectors corresponding to the plotted eigenvalues and some numerical simulations indicate that in the neighborhood of bifurcation points $\gamma=\gamma_{m}^{\text {opt }}$ also a type of maximum dissipation criterion is verified.

This optimization criterion indicates that the circuital resistance has to be chosen so as to locate the electro-mechanical poles at the first bifurcation points characterized by $\gamma_{m}^{\mathrm{opt}}$. In Fig. 25 the mechanical and electrical energy evolution of the dissipative PEM beam is shown using a too high, a too low and an optimal resistance respectively: referring to the beam specified by Table 3 it is estimated that the optimal resistance has the value of $29 \mathrm{k} \Omega$.

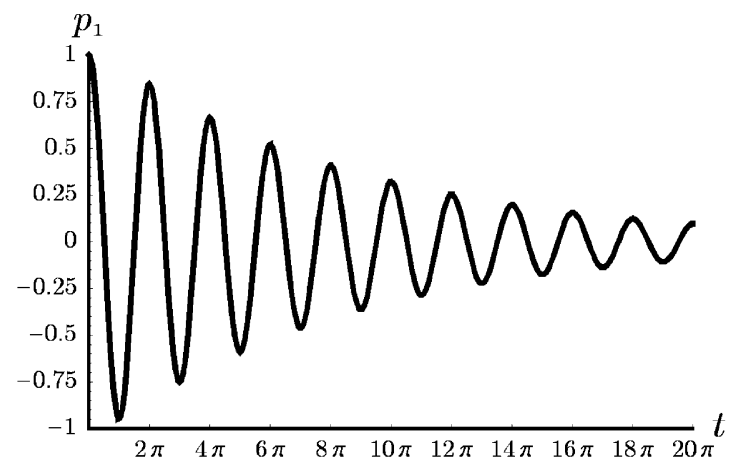

Fig. 24. Too low-shunt resistance time evolution. 

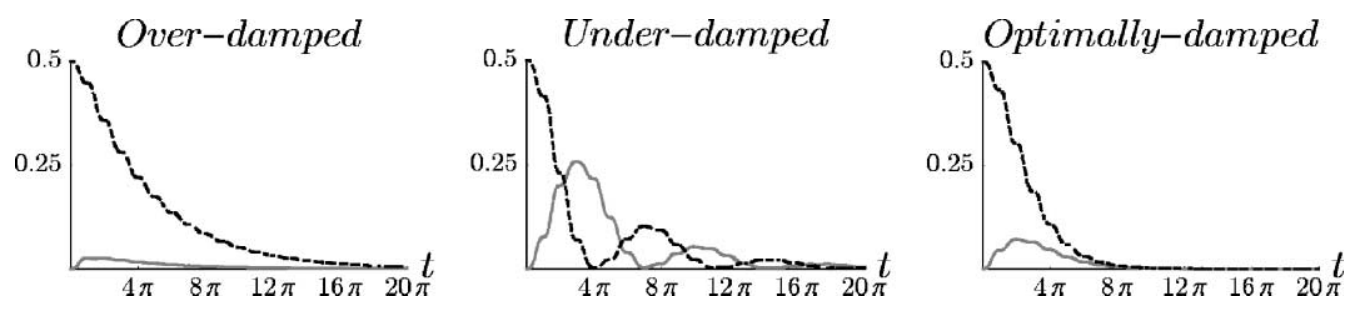

Fig. 25. Mechanical (dots) and electrical (solid) energy time evolutions.

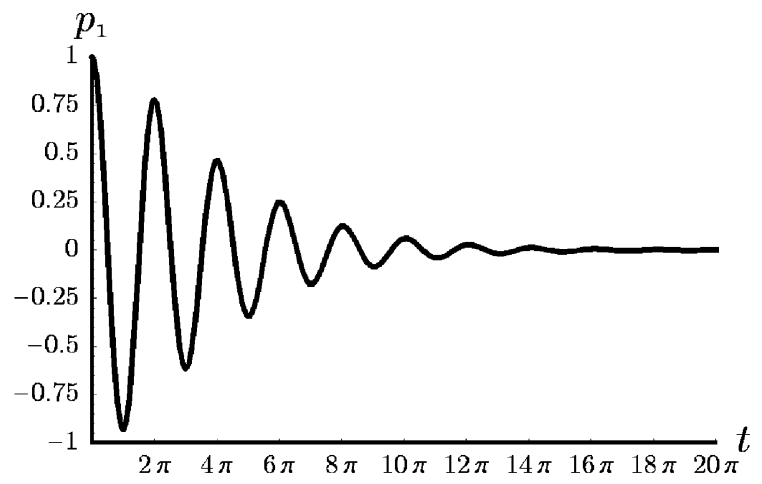

Fig. 26. Optimal damped evolution of the first mechanical coefficient.
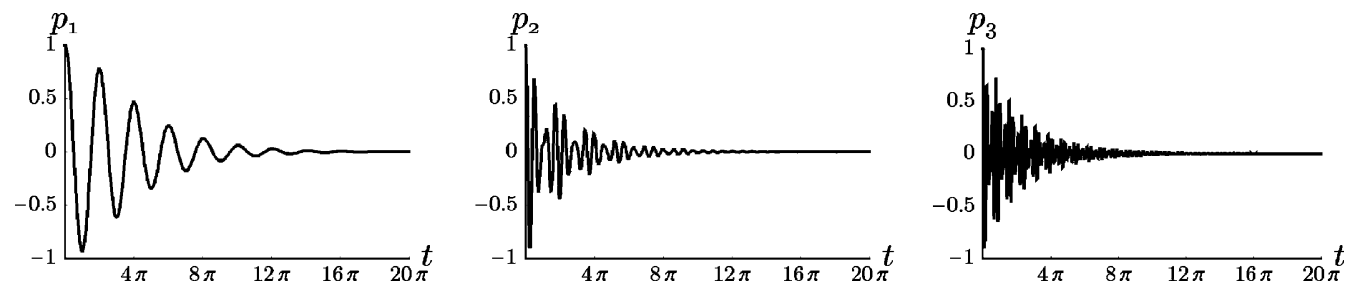

Fig. 27. Damped evolution of the first three modes.

In Fig. 26 the time evolution for the mechanical $p_{1}$ coefficient and for the related electro-mechanical energy content are shown, obtaining a damping ratio $\zeta_{1}^{\text {opt }}$ equal to $14.4 \%$.

In Fig. 27 the evolution of the first three mechanical modes is shown corresponding to purely mechanical initial data and with the value for resistance set equal to the optimal value relative to the first mode. As the eigenfrequency of the simply supported beam is an increasing function in dependence of the mode number, it is evident that when the first mode has been extinguished, also all harmonics are extinguished.

\section{PEM plate}

The results above obtained for the PEM beam can be simply generalized to a PEM plate. This is a useful by-product of the choice made when a PEM structure was defined using an "analog" electric circuit: indeed as a consequence of their definition all vibration problems for PEM structures reduce to a two-modes 
coupled evolution governed by an equation of the type (35). In the following the PEM plate is introduced and analyzed, exploiting an analog circuit synthesized from the finite-differences method approach, addressed in Section 2.

The concepts of the PEM beam and the PEM plate allow for the design of PEM frames, developed to control and damp mechanical vibrations in generic frames constituted by beams and plates. Let us explicitly remark that, generalizing the results exhibited in Section 2, the boundary conditions for the plate have a simple electric analog so that the electrical circuit exploited in the generic PEM frame is completely analog to the vibrating frame.

\subsection{Governing equation for the plate}

In order to derive the analog circuit for the plate, the dimensionless Laplace transform of the KirchhoffLove plate equation needs to be recalled. Let us consider a square plate of edge length $l$, thickness $h$, massdensity per unit area $\rho$ and the bending stiffness $S_{p}$, the governing equation for the deflection velocity $v$ is:

$$
\nabla^{2} \nabla^{2} \tilde{v}(\mathbf{r}, s)+\alpha^{4} s^{2} \tilde{v}(\mathbf{r}, s)=0, \quad \alpha^{4}=\varpi^{2} l^{4} \frac{\rho}{S_{p}},
$$

(the symbol $\nabla^{2} \nabla^{2}$ represent the biharmonic dimensionless operator and $\tilde{v}$ the deflection velocity Laplace transform). ${ }^{7}$

\subsection{Synthesis of the plate analog circuit}

Going through the steps taken in Section 2 to derive the beam analog based on the finite difference method, the plate analog circuit is easily derived from the two dimensions centered finite-differences approximation of Eq. (39) defined on a uniform-step grid sampling the spatial domain of the plate (see Fig. 28):

$$
\begin{aligned}
& \left(\frac{16}{\delta^{4}}+\alpha^{4} s^{2}\right) \tilde{v}_{i j}+\frac{\tilde{v}_{i+2 j}-6 \tilde{v}_{i+1 j}-6 \tilde{v}_{i-1 j}+\tilde{v}_{i-2 j}}{\delta^{4}}+\frac{\tilde{v}_{i j+2}-6 \tilde{v}_{i j+1}-6 \tilde{v}_{i j-1}+\tilde{v}_{i j-2}}{\delta^{4}} \\
& \quad+\frac{\tilde{v}_{i+1 j+1}+\tilde{v}_{i-1 j+1}+\tilde{v}_{i+1 j-1}+\tilde{v}_{i-1 j-1}}{\delta^{4}}=0 .
\end{aligned}
$$

Associating each sampling node of the grid to an electrical node of the analog circuit, Eq. (40) can be regarded as the governing equations for the electric potential of the circuit having the structure shown in Fig. 29, where

$$
L_{1}=L / 6, \quad L_{2}=L_{3}=-L
$$

and

$$
L C=\frac{\rho}{S_{p}}(\delta l)^{4} .
$$

Again, the presence of negative inductances in the analog circuit forbids its completely passive realization. However the considerations about the simulation of negative impedances provided in Section 2 still hold.

Let us underline that the plate circuital analog is not just the superposition of two beam analogs along the axial directions, since two additional transverse transmission lines are necessary.

\footnotetext{
${ }^{7}$ We choose $l$, a given fixed modal frequency $\varpi$ and $h$ as the characteristic constants for the dimensionless referential position vector r, Laplace-variable $s$ and deflection $u$ respectively.
} 


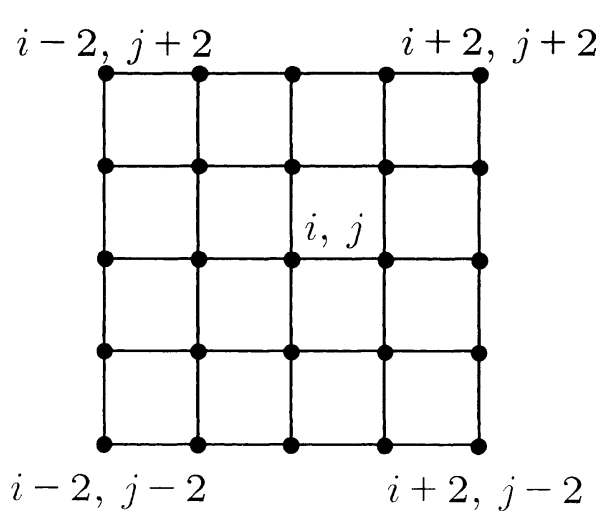

Fig. 28. Plate sampling grid.

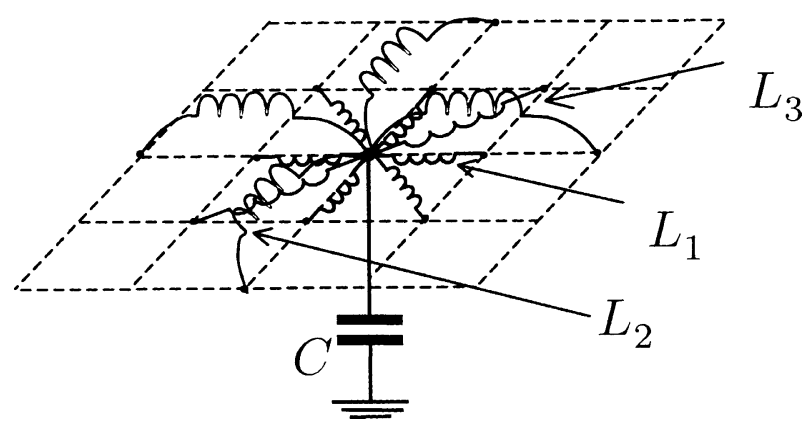

Fig. 29. Plate analog circuital module.

\subsection{Conservative PEM plate}

In analogy with the PEM beam we consider a plate on which a set of piezoelectric actuators are uniformly distributed and properly included in the plate circuital analog in order to realize the so called PEM plate. Again the actuators play the role of the capacitors connected between each electric node and the ground. Uniformly distributed square actuators are chosen to cover the plate, the influence factor $\eta$ is defined as the ratio between the area of the plate covered by the piezo layer and the area of the whole plate. Skipping the calculations, formally analogous to the ones detailed for the PEM beam, once the homogenization procedure for the electric quantities is considered, the following dimensionless governing equations for the PEM plate are found:

$$
\left[\begin{array} { l } 
{ \nabla ^ { 2 } \nabla ^ { 2 } v + \alpha ^ { 4 } \ddot { v } + \beta ^ { 2 } \nabla ^ { 2 } \dot { V } = 0 , } \\
{ \nabla ^ { 2 } \nabla ^ { 2 } V + \alpha ^ { 4 } \ddot { V } - \beta ^ { 2 } \nabla ^ { 2 } \dot { v } = 0 , }
\end{array} \left[\begin{array}{l}
\alpha^{4}=\varpi^{2} l^{4} \frac{\rho}{S_{p}}, \\
\beta^{2}=\frac{l^{2} \varpi g_{\mathrm{me}}}{S_{p}} \sqrt{\frac{\eta \rho}{k_{\mathrm{ee}}}},
\end{array}\right.\right.
$$

where $V$ is the dimensionless capacitive potential drop field, and the characteristic potential

$$
V_{0}=h \varpi \delta l \sqrt{\frac{\rho}{k_{\mathrm{ee}}}}
$$

was introduced. 
Considering as usual an expansion of the kinematical electrical and mechanical descriptors on the chosen set of basis functions, i.e.:

$$
\left[\begin{array}{l}
u(x, y, t) \\
\psi(x, y, t)
\end{array}\right]=\sum_{m=1}^{\infty} \sum_{n=1}^{\infty}\left[\begin{array}{l}
p_{m n}(t) \\
q_{m n}(t)
\end{array}\right] \varphi_{m n}(x, y),
$$

the following set of ODEs for the time-evolution for the coupled electro-mechanical modes of a simply supported PEM plate is achieved:

$$
\overline{\mathrm{d} t}\left[\begin{array}{c}
p_{m n}(t) \\
q_{m n}(t) \\
\dot{p}_{m n}(t) \\
\dot{q}_{m n}(t)
\end{array}\right]=\mathbf{A}_{m}\left[\begin{array}{c}
p_{m n}(t) \\
q_{m n}(t) \\
\dot{p}_{m n}(t) \\
\dot{q}_{m n}(t)
\end{array}\right],
$$

where $p_{m n}(t)$ and $q_{m n}(t)$ are the $m n$ mode Fourier coefficients and the evolution matrix is given by:

$$
\mathbf{A}_{m}=\left[\begin{array}{cccc}
0 & 0 & 1 & 0 \\
0 & 0 & 0 & 1 \\
-\frac{\pi^{4}\left(m^{2}+n^{2}\right)^{2}}{\alpha^{4}} & 0 & 0 & \frac{\pi^{2} \beta^{2}\left(m^{2}+n^{2}\right)}{\alpha^{4}} \\
0 & -\frac{\pi^{4}\left(m^{2}+n^{2}\right)^{2}}{\alpha^{4}} & -\frac{\pi^{2} \beta^{2}\left(m^{2}+n^{2}\right)}{\alpha^{4}} & -\frac{\gamma}{\alpha^{4}}
\end{array}\right] .
$$

The previous normal system is formally identical to (35) exhaustively analyzed in Section 2 dealing with the vibrations of a simply supported dissipative PEM beam and all qualitative considerations there developed hold.

Finally we consider a completely clamped aluminum PEM plate (its electro-mechanical characteristics are specified in Table 5) and find its electro-mechanical optimally damped vibrations induced by an initial impulse as specified in Fig. 32.

From Table 5, Eqs. (42) and (41) it is immediate to find the following valus for the inductors used in the analog circuit in Fig. 29:

$$
\left\{\begin{array}{l}
L_{1}=1.07 H \\
L_{2}=L_{3}=-6.44 H
\end{array}\right.
$$

We use again a Galerkin method; however, for computational reasons, instead of using the complex eigenmodes relative to the completely clamped plate equations we use the basis:

$$
\varphi_{m n}(x, y)=f_{m}(x) f_{n}(y), \quad n, m \in \mathbb{N},
$$

Table 5

Electro-mechanical parameters of the considered PEM plate

$\begin{array}{ll}\text { Edge length } & l=1 \mathrm{~m} \\ \text { Thickness } & h=4 \mathrm{~mm} \\ \text { Mass density per unit area } & \rho=10.8 \mathrm{~kg} / \mathrm{m}^{2} \\ \text { Bending stiffness } & S_{p}=410.3 \mathrm{Nm} \\ \text { PZT capacitance } & k_{\mathrm{ee}}=0.41 \times 10^{-6} \mathrm{~F} \\ \text { PZT coupling coefficient } & k_{\mathrm{me}}=3.55 \times 10^{-3}(\mathrm{~N} / \mathrm{V}) \mathrm{m}=\mathrm{C} \\ \text { PZT covering factor } & \eta=0.2 \\ \text { Number of actuators } & 100 \\ \text { Characteristic frequency } & \varpi=2\left(\pi^{2} / l^{2}\right) \sqrt{S_{p} / \rho}=121-7 \mathrm{rad} / \mathrm{s} \\ \text { Characteristic tension } & V_{0}=250 \mathrm{~V}\end{array}$


where

$f_{n}(\xi)=\cosh \left(\kappa_{n} \xi\right)-\cos \left(\kappa_{n} \xi\right)+\frac{\cosh \left(\kappa_{n} \xi\right)-\cos \left(\kappa_{n} \xi\right)}{\sinh \left(\kappa_{n} \xi\right)-\sin \left(\kappa_{n} \xi\right)}\left[\sin \left(\kappa_{n} \xi\right)-\sinh \left(\kappa_{n} \xi\right)\right]$
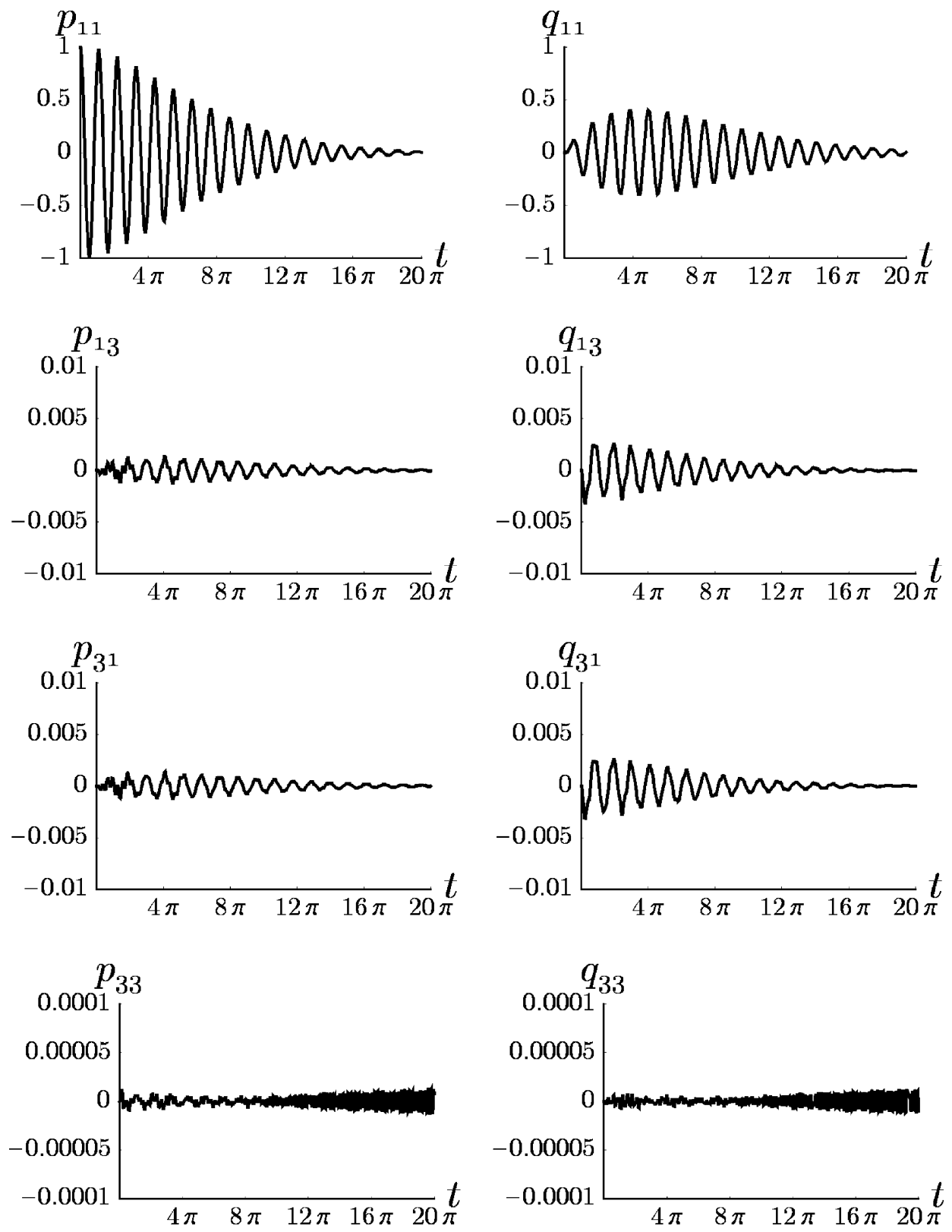

Fig. 31. Time evolution of the first non-vanishing electrical and mechanical modes. 
The analysis developed in the previous sections shows that the available piezoelectric transducers allow for a rapid damping of mechanical vibration in the case of structural members like those constituting a robotic arm, a panel for noise control or a helicopter rotor blade: indeed numerical simulations performed for PEM beams and plates are relative to commercial actuators, electric circuits which can be easily and cheaply realized, and aluminum members the larger dimensions of which are of the order of the meter.

Future theoretical investigations should include:

1. the study of more complex structures constituted by several beams and plates,

2. the conception of PEM shells and the determination of their evolution equations,

3. the study of the damping effect introduced by electric dissipation on forced vibrations of PEM structures.

Finally we underline that the theoretical results presented in this paper prove that an experimental prototype of PEM beams and plates can be detailedly designed and that the available technological state of the art finally allows for the engineering application of the concept of purely electrical and truly passive damping of mechanical vibrations exploiting PZT transducers. The construction of such a prototype will require, however, a main experimental effort.

\section{References}

Active Control eXperts (ACX), Inc., Cambridge, Massachussets, web site: www.acx.com.

Alessandroni, S., 2000. Master Thesis: Electrical analogs for plate equations and their applications in mechanical vibration suppression by P.Z.T. actuators. Virginia Tech.

Balanis, C.A., 1997. Antenna Theory: Analysis and Design. Wiley, New York.

Bardati, F., Barzilai, G., Gerosa, G., 1968. Elastic wave excitation in PZT slabs. IEEE Trans. Son. Ultrason. SU-15 (4), $193-202$.

Barzilai, G., Gerosa, G., 1967. Propagation of waves in special media and geometries. Final Scientific report Contract AF 61 (052)-807 6 Nov 1967.

Batra, R.C., Ghosh, K., 1995. Deflection control during dynamic deformations of a rectangular plate using piezoceramic elements. AIAA J. 33 (8), 1547-1549.

Bernadou, M., Haenel, C., submitted. Modelization and numerical approximation of piezoelectric thin shells Part 1, 2, 3, submitted for publication.

Bernadou, M., Haenel, C., 2000. Modelization and numerical analysis of active thin shell structures. In: Proceedings European Congress on Computational Methods in Applied Sciences and Engineering EECOMAS 2000 Barcelona.

Botkin, N.D., 1999. Homogenization of an equation describing linear thin plates excited by piezopatches communications. Appl. Anal. 3 (2), 271-281.

Brillouin, L., 1946. Wave Propagation in Periodic Structures, Electric filters and Crystal Lattices. McGraw-Hill, New York.

Bruton, L.T., 1980. RC-active Circuits: Theory and Design. Prentice-Hall, Englewood Cliffs, NJ.

Crandall, S.H., Karnopp, D.C., Kurtz, E.F., Pridmore-Brown, D.C., 1968. Dynamics of Mechanical and Electromechanical Systems. McGraw-Hill, New York.

D'Ambrogio, W., Sestieri, A., 1989. A modification method for vibration control of structures. Mech. Syst. Signal Process. 3, $229-253$.

dell'Isola, F., Vidoli, S., 1998a. Continuum modelling of piezo-electro-mechanical truss beams: an application to vibration damping. Arch. Appl. Mech. 68 (1), 1-19.

dell'Isola, F., Vidoli, S., 1998b. Bending-waves damping in truss beams by electrical transmission line with PZT actuators. Arch. Appl. Mech. 68, 626-636.

Firestone, F.A., 1956. Twixt earth and sky with rod and tube; the mobility and classical impedance analogies. J. Acoust. Soc. Am. 28 (6), 1117-1153.

Gandhi, M.V., Thompson, B.S., 1992. Smart Materials and Structures. Chapman and Hall, London.

Hagood, N.W., von Flotow, A.H., 1991. Damping of structural vibrations with piezoelectric materials and passive electrical networks. J. Sound Vib. 146 (2), 243-268.

Hoffmann, K.H., Botkin, N.D., 1999. Homogenization of a model describing vibration of nonlinear thin plates excited by piezopatches. Int. Ser. Numer. Math. 133, 191-200.

Hoffmann, K.H., Botkin, N.D., 2000. Homogenization of von Karman plates excited by piezoelectric patches. ZAMM Z. Angew. Math. Mech. 80 (9), 579-590. 
Inman D., 2000. Lecture notes for ME 5984: Smart Structures, Virginia Tech.

Karplus, W.J., Soroka, W.W., 1959. Analog Methods, Computation and Simulation. McGraw-Hill, New York.

Molloy, C.T., 1958. Four pole parameters in vibration analysis in mechanical impedance methods for mechanical vibrations. In: Plunkett, R. (Ed.), Colloquium on Mechanical Impedance Methods for Mechanical Vibrations presented at the ASME Annual Meeting, New York, NY, December 2, sponsored by Shock and Vibrating Committee Applied Mechanics Division, The American Society of Mechanical Engineers, New York.

Newcomb, R.W., 1966. Linear Multiport Synthesis. McGraw-Hill, New York.

Papoulis, A., 1991. Probability, Random Variables, and Stochastic Processes. McGraw-Hill, New York.

Piezo Systems, Inc., Cambridge, Massachussets, web site: www.piezo.com.

Porfiri, M., 2000. Master Thesis: Synthesis of electrical networks interconnecting PZT actuators to efficiently damp mechanical vibrations, Virginia Tech.

Rogacheva, N.N., 1994. The Theory of Piezoelectric Shells and Plates. CRC Press, Boca Raton.

Valis, T., vonFlotow, A.H., Hagood, N.W., 1992. An acousto-electromagnetic piezoelectric waveguide-couple. In: Active Materials and Adaptive Structures Proceedings of the ADPA/AIAA/ASME/SPIE Conference Nov. 1991 Alexandria Virginia Gareth J Knowles Ed. Inst. of Physics Publishing Bristol and Philadelphia, pp. 383-394.

Vidoli, S., dell'Isola, F., 2000. Modal coupling in one-dimensional electro-mechanical structured continua. Acta Mechanica 141, 1-2.

Wang, K.W., 1995. Structural vibration suppression via parametric control actions piezoelectric materials with real-time semi-active networks. In: Guran, A., Inman, D.J. (Eds.), Wave Motion, Intelligent Structures and Nonlinear Mechanics, Series on Stability, Vibration and Control of Structures: vol. 1, World Scientific Singapore, pp. 112-134. 